RESEARCH LETTER - Professional Development

\title{
Optimizing Small World Initiative service learning by focusing on antibiotics-producing actinomycetes from
} soil

\author{
Piet W. J. de Groot ${ }^{1,2,3,4, *, \dagger}$, Jordan Fernández-Pereira ${ }^{2,3}$, Rosario Sabariegos ${ }^{2,3}$, \\ Pilar Clemente-Casares ${ }^{1,3}$, Javier Parra-Martínez ${ }^{5}$, Víctor J. Cid ${ }^{6}$ and Diego
}

\section{A. Moreno $0^{1,7, \dagger}$}

${ }^{1}$ Universidad de Castilla-La Mancha, Facultad de Farmacia, Avda. Dr. José María Sánchez Ibáñez s/n, E-02008

Albacete, Spain, ${ }^{2}$ Universidad de Castilla-La Mancha, Facultad de Medicina, c/ Almansa 14, E-02008 Albacete, Spain, ${ }^{3}$ Universidad de Castilla-La Mancha, Centro Regional de Investigaciones Biomédicas, c/ Almansa 14, E-02008 Albacete, Spain, ${ }^{4}$ Fundación Parque Científico y Tecnológico de Castilla-La Mancha, Paseo de la Innovación 1, E-02006 Albacete, Spain, ${ }^{5}$ Complejo Hospitalario Universitario de Albacete (CHUA), Servicio de Microbiología, c/ Hermanos Falcó 37, E-02006 Albacete, Spain, ${ }^{6}$ Universidad Complutense de Madrid, Departamento de Microbiología y Parasitología, Facultad de Farmacia, Pza. Ramón y Cajal s/n. E-28040, Madrid, Spain and ${ }^{7}$ Universidad Politécnica de Madrid, Escuela Técnica Superior de Ingenieros Industriales, c/ José Gutiérrez Abascal 2, E-28006 Madrid, Spain

${ }^{*}$ Corresponding author: Centro Regional de Investigaciones Biomédicas, Universidad de Castilla-La Mancha, Calle Almansa 14, E-02008 Albacete, Spain. Tel: +34-967599200-2889; Fax: +34-967599360; E-mail: piet.degroot@uclm.es

One sentence summary: Optimization of Small World Initiative citizen science and service learning by focusing on isolation of antibiotic-producing actinomycetes from soil.

\begin{abstract}
Small World Initiative and Tiny Earth are popular citizen science programs that are implemented worldwide in response to the global antibiotic resistance crisis. When starting up the program in Albacete (Spain), we noted that rates of isolated antibiotic-producing bacteria are generally low. To make the activity more stimulating for participating students, we modified the protocol to obtain more positive results by focusing on isolation of actinomycetes, the main producers of most clinically used antibiotics. Adaptations involved redesigning culture media, incubation times and temperatures, and modification of the ESKAPE antibiosis experiment by employing an agar-transplantation step. Of 390 bacterial isolates tested, almost $6 \%$ tested positive in antibiosis experiments and DNA sequence analysis confirmed that all positives are actinomycetes, demonstrating that our protocol is efficient toward isolating antibiotic-producing actinomycetes from soil. Evaluation forms filled by participating students indicated that the program was received very positively and that our modifications contribute to make this educational program more stimulating and efficient.
\end{abstract}




\section{INTRODUCTION}

The popular educational citizen science (also named student sourcing) programs Small World Initiative (SWI; http://www.smallworldinitiative.org/) and Tiny Earth (TE, http://tinyearth.wisc.edu/; Hernandez et al. 2018), originally setup and developed at Yale University and the University of Wisconsin-Madison, aim at increasing societal awareness of the danger of improper use of antibiotics by teaching high school students on the growing global antimicrobial resistance (AMR) problem. The programs' intent is to awake the students' interest in the topic and to trigger their citizenship by letting them participate in a real experiment that involves a screening to isolate novel antibiotic-producing microorganisms from self-taken soil samples. In lectures and tutorials, in addition to details of the experiment, the scholars receive education on AMR and the use of antibiotics. They are also stimulated to discuss the topic in their home environment. Thus, apart from enabling a large-scale search for new antibiotic-producing bacteria by the participation of a large group of young, motivated students, objectives of the SWI and TE programs are to achieve a better awareness in human society of the antibiotic resistance problem, to stimulate the scientific interest and motivation of high school students by letting them participate in an exciting and relevant science project and to improve dropout numbers among these students. Due to its success, SWI/TE citizen science has rapidly expanded in the USA, and is also implemented in many other countries around the globe (Caruso et al. 2016).

In SWI@Spain, recently renamed MicroMundo, promoted by researchers of the Spanish Society for Microbiology (SEM), 31 nodes at many different universities and research institutes in Spain and Portugal are running the program in a servicelearning setup (Valderrama et al. 2018). Service learning is defined as an educational experience in which students participate in the activity of a community to develop knowledge on a topic while acquiring civic awareness (Bringle and Hatcher 1996). Service-learning projects can be carried out in different areas such as geology, chemistry, environment and biology (Abrahamsen 2004). Reviews and meta-analysis of servicelearning programs in clinical microbiology reported benefits for participating undergraduate students in self-attitude, education and academic achievement, civil engagement, social skills and moral reasoning (Stewart and Wubbena 2014; Webb 2017). In the SWI@Spain/MicroMundo program, undergraduate students act as SWI teaching assistants (SWITAs), thus involving two educational levels (higher education and high school) with different degrees of responsibilities. In training sessions, SWITAs learn about the topic, practice key experimental aspects, get teaching instructions and prepare and practice high school lectures, and learn to evaluate and discuss results. At the high schools, the SWITAs provide teaching on the AMR problem, discuss with the scholars about the topic, explain and guide the scholars through their experiments and discuss the results (Valderrama et al. 2018). Thus, the service-learning setup has additive value in that it provides an excellent opportunity for undergraduate students to get some first teaching experience in a real high school environment while triggering their scientific interest by managing a challenging science project in close contact with faculty members.

In the last decades, the number of multidrug resistant bacteria considered as a serious threat to human health has increased progressively (Leung et al. 2011). Inappropriate or excessive use of antibiotics has contributed to the problem and is still an important issue (Cantón and Morosini 2011). Few antibiotics have been launched recently, and the compounds that are currently in clinical trials will not be sufficient to tackle the impending AMR threat (Butler, Blaskovich and Cooper 2017). Most antibiotics used in the clinic and most new antibiotics from natural products originate from actinomycetes. However, when starting up the SWI@Spain/MicroMundo program in Albacete at the University of Castilla-La Mancha (UCLM), we noted that the original SWI protocol is not specifically designed for finding actinomycetes (DeLuca, King and Morsy 2017) and that the success rate of isolating antibiotic-producing bacteria is rather low. We hypothesized that a higher success rate might help to create a more stimulating educational atmosphere on both teaching levels, for the undergraduate SWITAs as well as the high school students. Therefore, with the aim of optimizing the educational efficiency of the SWI/TE program as well as its potential scientific outcome, we have implemented important methodological adaptations to the SWI protocol to focus the experiment more on actinomycetes. Student perception of the program and learning outcomes were evaluated through anonymous questionnaires after completion of the activity.

\section{MATERIALS AND METHODS}

\section{SWI-Albacete service-learning setup and SWITA training sessions}

SWI-Albacete was carried out as an extracurricular servicelearning activity by 26 undergraduate university students with 75 participating scholars from four high schools in Albacete. One UCLM staff member coordinated the program with four additional university teachers being responsible for directing the activity in the participating high schools. This setup is similar to the SWI@Spain/MicroMundo pilot, detailed in Valderrama et al. (2018). The undergraduates were students from the Faculties of Medicine and Pharmacy (13 each), between second and fifth grade. Students were mixed to create four equally experienced teams. Because of their dense teaching schedules, students were not asked to prepare media and materials for high school sessions.

SWITAs were prepared for their teaching tasks during training sessions. They first received education on AMR, (im)proper use of antibiotics, laboratory safety and soil sampling. As many of the SWITAs had not yet followed any practical microbiology course prior to the program, two training sessions were focused mainly on getting the SWITAs acquainted with the experimental methodology, including the new setup of the antibiosis experiment and how to recognize actinomycetes. With examples of tutorials, SWITAs were prepared for giving classes at high schools and instructed to prepare and practice their lectures/tutorials.

\section{Projected learning outcomes}

Learning outcomes for the high school students are expected to include: (i) improved awareness of the AMR problem; (ii) improved knowledge on the proper use of antibiotics; (iii) awareness of the difficulty of antibiotic discovery; (iv) receiving basic 
scientific training in the field of microbiology; $(v)$ raising scientific interest; (vi) learning to deal with failure/frustration of unsuccessful experimentation; and (vii) working in a collaborative environment. For the SWITAs, additional learning outcomes include (viii) obtaining teaching experience in a real high school setting; (ix) managing preparation of tutorials for the classes, (x) functioning as a team player; and (xi) divulgation.

\section{Safety measures}

A microbial teaching activity with unexperienced scholars requires special safety procedures. However, participating high schools do not need specialized microbiology laboratories with biosafety rating. Because all high school students were $<18$ years old, all activities and data collection were approved by the school councils and their parents via signed statements. Students were instructed to follow biosafety guidelines for handling microorganisms in a teaching laboratory (Emmert 2013). At the end of each session, the laboratory was cleaned up and lab benches disinfected (also before the sessions) by the SWITAs. Transfer of any material or equipment outside the classroom was performed by the faculty staff, who were also responsible for incubation and storage of inoculated plates at the university.

\section{Bacterial strains, culture media and growth conditions}

Dilution series of soil samples were plated on Actinomycete Isolation (AI; Merck, Madrid, Spain) agar or R2A (Oxoid, Madrid, Spain) agar. The AI medium is supplemented with $5 \mathrm{~mL} / \mathrm{L}$ glycerol (Panreac, Castellar del Vallès, Spain). Agar concentrations in both media were adjusted to $2 \%(\mathrm{w} / \mathrm{v})$. Antimicrobials dieldrin $(4 \mathrm{mg} / \mathrm{L})$, nalidixic acid $(20 \mathrm{mg} / \mathrm{L})$ and cycloheximide $(80 \mathrm{mg} / \mathrm{L})$, all from Merck, were added to inhibit growth of mites, Gramnegative bacteria and fungi, respectively. Selected individual colonies were grown on the same media without antibiotics.

Antibiosis (ESKAPE) experiments were performed on tryptic soy broth (Oxoid) agar (TSA) plates. The acronym ESKAPE includes six leading nosocomial pathogens that exhibit multidrug resistance and virulence: Enterococcus faecium, Staphylococcus aureus, Klebsiella pneumoniae, Acinetobacter baumannii, Pseudomonas aeruginosa and Enterobacter spp. In the SWI program, harmless ESKAPE relatives are used. ESKAPE relatives Bacillus subtilis ATCC 6051, Staphylococcus epidermidis ATCC 14990, Escherichia coli ATCC 11775, Acinetobacter baylyi ATCC 33305, Pseudomonas putida ATCC 12633 and Enterobacter aerogenes ATCC 51697 were maintained on TSA and grown overnight at $37^{\circ} \mathrm{C}$ and $250 \mathrm{rpm}$ in tryptic soy broth for the antibiosis experiment.

\section{SWI high school sessions}

At the high schools, the program consisted of five sessions (Fig. 1). In session 1, first an extensive introductory lecture of $\sim 1 \mathrm{~h}$ was given by SWITAs on AMR and application of antibiotics. Scholars were stimulated to discuss the topic at home with friends and family. Theory and experimental details of the SWI experiment were outlined, and sampling instructions were given and demonstrated in the school grounds. Each pair of scholars received a soil isolation kit consisting of gloves, a sterile spatula and a sterile $50 \mathrm{~mL}$ Falcon tube in a closed plastic bag.

Samples were collected by scholars in different places in the provinces Albacete and Cuenca (Table S1, Supporting Information) and stored in a cool place until use. The Nagoya Protocol for protection of the biodiversity in the area where samples were taken was followed: samples were encoded, their geolocalizations recorded and photographs of the sites were taken.

The following sessions each started with a brief 20-25 min tutorial by SWITAs outlining the experimental details of the respective sessions. In session 2, samples containing 2-3 g of soil were mixed with sterile MilliQ water $(10 \mathrm{~mL} / \mathrm{g}$ soil) by vortexing for $1 \mathrm{~min}$. Ten-fold serial dilutions were prepared and plated on AI or R2A agar containing antibiotics to promote enrichment of actinomycetes. Plates were incubated for 10 days at $28^{\circ} \mathrm{C}$ to allow for growth of slow-growing actinomycetes.

In session 3, the number of colony forming units per gram soil (CFU/g) in each sample was determined (Table S1, Supporting Information). Then, each scholar pair selected at least 10 candidate colonies and recorded their morphologies. Potentially interesting actinomycete colonies were selected based on criteria such as invasive growth, filamentous appearance, nonsmooth morphology, being in an isolated location on the plate and inhibiting growth of neighboring colonies. Selected colonies were resuspended in sterile MilliQ water and streaked in the shape of an H (Fig. 1) on AI or R2A agar (without antimicrobials) with a sterile cotton swab. Plates were again incubated for $\sim 10$ days at $28^{\circ} \mathrm{C}$.

In session 4, all scholar pairs checked antagonistic activity of 10 isolates against 6 different ESKAPE relatives. First, 100 $\mu \mathrm{L}$ of pre-grown ESKAPE-relative cultures were seeded on separate TSA plates and allowed to dry. Then, using Transfertubes (Repligen, Breda, The Netherlands), agar disks adjacent to grown selected candidate actinomycete bacteria were deposited clockwise on each of the seeded TSA plates. A filter disk containing $30 \mu \mathrm{g}$ of chloramphenicol (Oxoid) was placed in the center of the plates as a positive control. Plates were incubated for $24 \mathrm{~h}$ at $37^{\circ} \mathrm{C}$, photographed and stored at $4^{\circ} \mathrm{C}$. In session 5 , antagonistic activity of bacteria was assessed by the presence of a transparent zone (halo) around the deposited agar disks and halo sizes (diameter) were measured. Results were discussed with the whole class and further clarified by SWITAs in a tutorial.

\section{Evaluation of SWI service learning and divulgation}

Perception of the SWI program by the participating scholars and SWITAs and achievement of learning goals were assessed through an anonymous questionnaire after completion of the activity. The questionnaire comprised 11 questions (detailed in Fig. 2) with response options in a Likert-type scale ranging from 1 (strongly disagree) to 5 (strongly agree) and 3 open-answer questions to indicate what they considered the best, worst or improvable aspects of the program. The questionnaire for the undergraduates included five additional questions about SWITA training sessions and service-learning aspects.

SWI-Albacete also included divulgation activities by SWITAs on Twitter (@AlbaceteSWI), interviews and articles in local newspapers (La Tribuna de Albacete, 24-Feb-2019; https://di ariosanitario.com/aprendices-fleming-swi/) and a closing ceremony during which the scholars and students received an SWI/MicroMundo-research diploma. Renowned speakers participated in the ceremony by giving divulgation lectures on AMR and antibiotic discovery, and one scholar and one SWITA gave short talks about their personal experience. Results of the program have been presented at the 17th National Microbiology Conference organized by SEM, and at the 3rd Conference on Teaching Innovation in Higher Education organized by UCLM. 

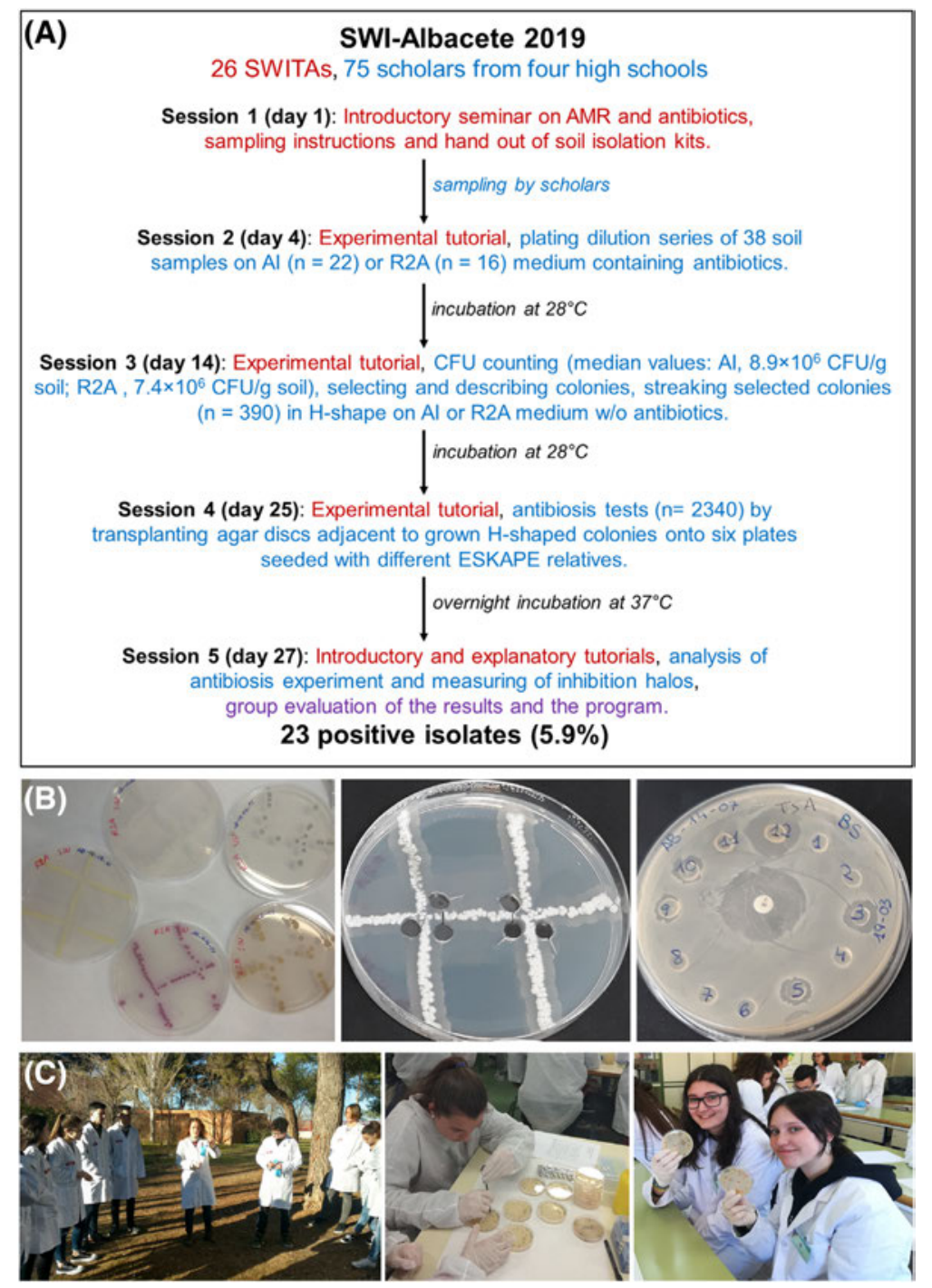

Figure 1. SWI-Albacete experimental protocol and examples. (A) Scheme of SWI-Albacete protocol. (B, left and center panels) Examples of selected colonies, inoculated in $\mathrm{H}$-shape and grown for 11 days at $28^{\circ} \mathrm{C}$, before (left) and after (center) transplanting agar disks. (B, right panel) Example of antibiosis experiment with ESKAPE-relative B. subt lis after $24 \mathrm{~h}$ at $37^{\circ} \mathrm{C}$. Agar disks adjacent to grown selected colonies were deposited on TSA plates seeded with an ESKAPE-relative. Controls include a filterpaper disk containing $30 \mu \mathrm{g}$ chloramphenicol (center of plate) and an agar disk from a TSA plate containing $50 \mu \mathrm{g} / \mathrm{mL} \mathrm{chloramphenicol} \mathrm{(top,} \mathrm{agar} \mathrm{disk} 12$ ). Antagonistic growth inhibition is visible as clear zones (halos) around agar disks. (C, left panel) SWITAs giving sampling instructions during high school session 1. (C, center panel) High school students performing the antibiosis experiment during session 4. (C, right panel) Students proudly showing results of their antibiosis test during evaluation session 5 .

\section{Taxonomic analysis}

To obtain DNA for taxonomic analysis, cell material was scraped from agar plates and lysed using Genejet (Thermo Fisher) solutions with addition of $10 \mathrm{mg} / \mathrm{mL}$ lysozyme instead of RNase in the first step. Upon cell lysis, samples were centrifuged at high speed for $10 \mathrm{~min}$, and DNA in the supernatants was precipitated with isopropanol. Washed and dried DNA pellets were taken up in TE (10 mM Tris-HCl, 1 mM EDTA, pH 8.0) buffer containing $50 \mu \mathrm{g} / \mathrm{mL}$ RNase A and incubated for $30 \mathrm{~min}$ at $37^{\circ} \mathrm{C}$. The samples were extracted with chloroform, DNA was precipitated with ethanol and finally taken up in $25 \mu \mathrm{L}$ TE buffer. Oligonucleotides 16S_339_F: 5'-CTCCTACGGGAGGCAGCAG-3' and 16S_1087_R: 5'-CTCGTTGCGGGACTTAACCC-3' for partial 16S rDNA PCR amplificationpt were designed, yielding amplicons of $\sim 0.75 \mathrm{~kb}$ that include variable regions V3-V5 of 16S rDNA (Weisburg et al. 1991). Colony PCR was performed with Taq polymerase
(EURx) using a standard PCR program. PCR fragments were cleaned up using Micro Bio-Spin P-30 columns (Bio-Rad) and sequenced (STAB-Vida). Taxonomic and phylogenetic DNA sequence analysis was performed using NCBI-Blast and MEGA $\mathrm{X}$ (Kumar et al. 2018) software.

\section{RESULTS AND DISCUSSION}

\section{Optimizing SWI/TE by focusing on actinomycetes}

The main objective of SWI/TE citizen science is to improve societal awareness of the growing AMR problem by running an experimental course with high school students that is aimed at discovering new antibiotic-producing bacteria. One of the setbacks of the original SWI/TE protocol is that only few students find positive isolates, which does not help to trigger their 
(A)

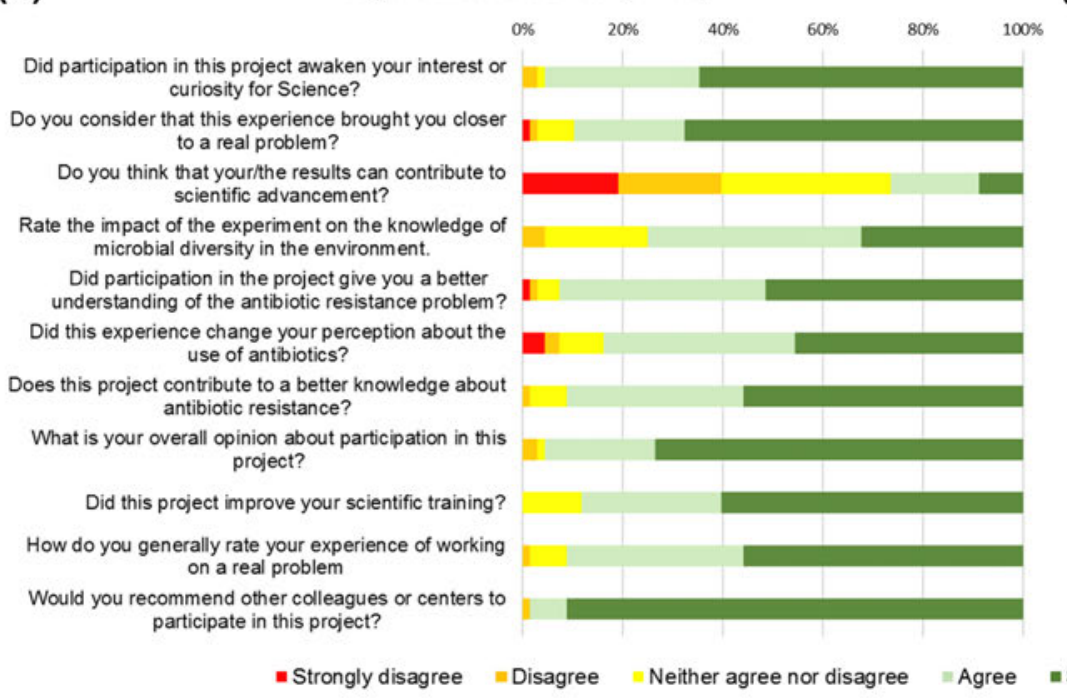

(B)

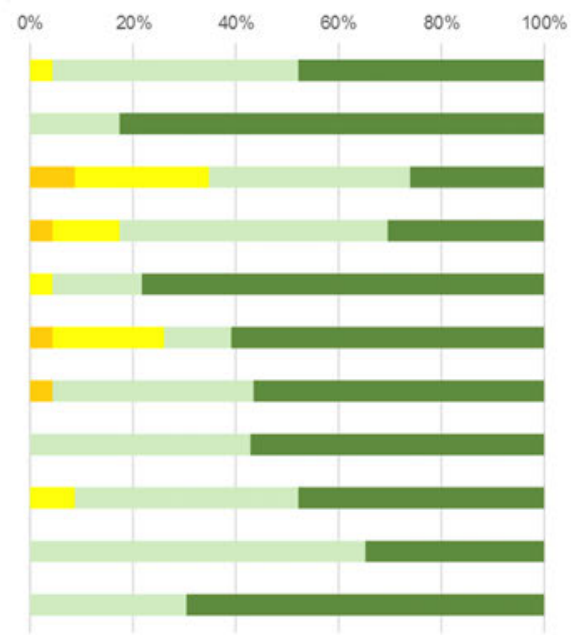

(C)

Training session questions SWITAs $(n=23)$

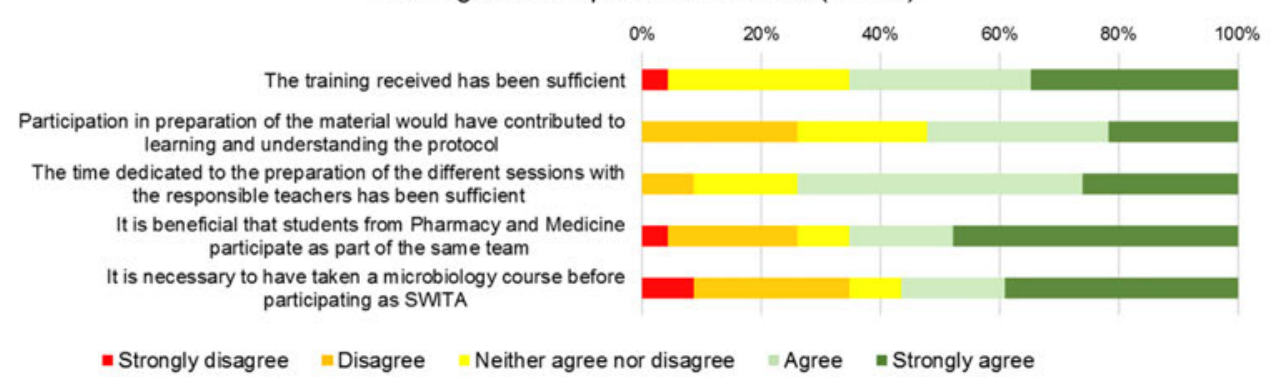

Figure 2. Student perception of the SWI service-learning program. Anonymous questionnaires with 11 questions about the SWI program with response options in a Likert-type scale were filled by high school students (A) and SWITAs (B). SWITAs were also asked to answer five questions about the SWITA training sessions (C).

interest in the topic or to make them think that they can contribute to scientific advancement of the field. The original protocol is not focused on isolating actinomycetes, known producers of most clinically used antibiotics. To make the SWI teaching program more stimulating and effective education wise as well as in a scientific sense, we (SWI-Albacete) made substantial modifications to the SWI protocol (Hernandez et al. 2018) in order to focus on the isolation of antibiotic-producing actinomycetes.

The modified procedure of SWI-Albacete is detailed in the Materials and Methods section and Fig. 1. Culture media, incubation times and temperatures of the original SWI@Spain protocol were adapted to favor growth of actinomycetes. Dilution series of the soil samples (see Table S1, Supporting Information, for sample details) were seeded on AI or R2A agar supplemented with cycloheximide, dieldrin and nalidixic acid (instead of $10 \%$ TSA supplemented with cycloheximide), and plates were incubated at $28^{\circ} \mathrm{C}$ for 10 days (instead of $20-24^{\circ} \mathrm{C}$ until colonies appeared on the plates). After the 10-day incubation period, many actinomycete-like colonies had grown on the AI and R2A plates. Selected potentially interesting colonies were streaked on fresh plates and allowed to grow and secrete potential antibiotic metabolites for 10-11 days. Importantly, in the final antibiosis-ESKAPE-experiment, instead of streaking selected colonies, agar disks from areas immediately adjacent to the grown colonies, potentially containing compounds with antagonistic activity, were transferred onto plates that were seeded with relatives of ESKAPE pathogens (Fig. 1). Of the 390 tested isolates, 23 isolates (5.9\%) showed inhibition halos for the Gram-positive bacteria B. subtilis and/or S. epidermidis (Table 1). This rate of positive isolates is more than 2fold higher compared to the original SWI@Spain pilot (Valderrama et al. 2018). Validation of the antibiosis results by university staff confirmed the 23 hits. No halos were observed on plates seeded with Gram-negative ESKAPE relatives. The reason that no activity against Gram-negative bacteria was detected is probably because of their cell wall outer membrane, which acts as a barrier to prevent entry of antibiotics into the cell (Butler and Cooper 2011). Consistent with this, the majority of the known antibiotics only act against Gram-positive bacteria (Berdy 2005) and worryingly few oral antibiotics for treatment of infections by Gram-negative pathogens are in the pipeline.

\section{Student perception and learning outcome of SWI-Albacete}

Analysis of evaluation questionnaires filled by the students showed that the appreciation of participating in SWI-Albacete was very high, both in the case of the high school students $(n=68 ; 96 \%)$ and the SWITAs $(n=23 ; 100 \%)$, and almost all 
TABLE 1 Isolated antibiotic-producing bacteria.

\begin{tabular}{|c|c|c|c|}
\hline \multirow[b]{2}{*}{ Sample SWI code } & \multicolumn{2}{|c|}{ Size of halo (mm) } & \multirow[b]{2}{*}{ NCBI-Blast result } \\
\hline & $\mathrm{Bs}^{\mathrm{a}}$ & $\mathrm{Se}^{\mathrm{a}}$ & \\
\hline AB-07-01-06 & 6 & 9.5 & $100 \%$ id. with S. spiroverticillatus, S. graminofaciens and S. cremeus \\
\hline AB-07-06-01 & 11 & 7 & $100 \%$ id. with S. iakyrus, S. bellus, S. coerulescens and S. coeruleorubidus ${ }^{b}$ \\
\hline AB-07-06-04 & 7.5 & 8 & Identical to AB-07-06-01 \\
\hline AB-07-06-08 & 9.5 & 8 & Identical to $\mathrm{AB}-07-06-01$ \\
\hline AB-07-06-09 & 11 & 10 & Identical to AB-07-06-01 \\
\hline AB-07-08-10 & 9.5 & 10 & $100 \%$ id. with S. hachijoensis, S. netropsis, S. eurocidicus and S. syringium \\
\hline AB-07-09-02 & 7.5 & 7.5 & $100 \%$ id. with S. canus \\
\hline AB-09-09-03 & 12.5 & 14 & $100 \%$ id. with S. lavendulocolor and S. luridus \\
\hline AB-14-04-01 & 8.5 & 7 & $100 \%$ id. with S. parvulus \\
\hline AB-14-06-01 & 7.5 & 6 & $100 \%$ id. with S. iakyrus, S. bellus, S. coerulescens and S. coeruleorubidus \\
\hline AB-14-06-04 & 7 & 6.5 & 99.58\% id. with various Streptomyces spp., e.g. S. swartbergensis and S. levis \\
\hline AB-14-06-07 & 11.5 & 11.5 & 100\% id. with various Streptomyces spp., e.g. S. hawaiiensis and S. janthinus \\
\hline AB-14-07-03 & 11.5 & 7 & $\begin{array}{l}99.86 \% \text { id with Amycolatopsis speibonae, A. thailandensis, A. umgeniensis } \\
\text { and A. coloradensis }\end{array}$ \\
\hline AB-14-07-05 & 10 & 8 & Identical to AB-14-06-07 \\
\hline AB-14-07-07 & 8.5 & 8.5 & Identical to AB-14-06-07 \\
\hline AB-14-07-09 & 9 & 6.5 & Identical to $\mathrm{AB}-14-06-07$ \\
\hline$A B-14-07-10$ & 10 & 9 & Identical to $\mathrm{AB}-14-06-07$ \\
\hline AB-14-08-01 & 7 & 6 & Identical to $\mathrm{AB}-14-06-01$ \\
\hline AB-14-09-01 & 7 & 7 & Identical to AB-14-06-01 \\
\hline AB-14-09-09 & 11 & 16 & 100\% id. with S. peucetius, S. xantholiticus and S. kurssanovii \\
\hline AB-99-01-08 & 15 & 15 & $100 \%$ id. with various Streptomyces spp., e.g. S. tendae and S. rutgersensis \\
\hline AB-99-04-03 & 8.5 & 15 & Identical to AB-14-09-09 \\
\hline AB-99-06-10 & 6 & 16 & $99.86 \%$ id. with S. rishiriensis \\
\hline
\end{tabular}

aBs, Bacillus subtilis; Se, Staphylococcus epidermidis.

${ }^{\mathrm{b}}$ Small ambiguity in $16 \mathrm{~S}$ rDNA sequence.

students in both groups would recommend the program to colleagues or centers (Fig. 2). As an example of their enthusiasm, several SWITAs have requested to also participate in SWIAlbacete 2020 and, as a consequence, $22 \%$ of the SWITAs in the new program will be repeaters. Having such experienced students in the new SWITA teams will present obvious advantages; these seniors can act as leaders of their teams, thus taking on a different level of responsibilities and duties. The SWI program evidently also increased awareness of both scholars (93\%) and SWITAs (96\%) of the global AMR problem, improved their knowledge, in particular of the scholars (84\%), on the correct use of antibiotics and both groups (scholars, 91\%; SWITAs, 100\%) also highly valued having been able to participate in a scientific experiment that is targeting a real problem. They also considered that the program has improved their scientific training (scholars, 88\%; SWITAs, 91\%). The scientific interest of SWITAs in microbiology was clearly raised as several have performed short summer internships in our laboratories after completing the activity, while some senior SWITAs have started end-ofgrade projects with microbiology teachers. Furthermore, several SWITAs voluntarily participated in European Antibiotic Awareness Day activities organized in the framework of the Spanish National Antibiotic Resistance Plan (PRAN; http://resistenci aantibioticos.es/es). The unexperienced second grade SWITAs received microbiology classes in semesters during or following the program. Without exceptions, all these SWITAs showed an active attitude in class and were scoring among the best students of their year. Thus, at least in the field of microbiology, participation in the program had a very positive effect on the students. The enthusiasm of the SWITAs for this servicelearning activity was also notable by their active posting on
Twitter (@AlbaceteSWI) about their progress or photos taken during teaching sessions.

To the question if they think that their results will contribute to scientific advancement, not more than $26 \%$ of the high school students responded positively. This is consistent with the fact that most scholars (67\%) had not identified positive hits despite the overall higher rate of positive isolates. Among the SWITAs the response to this question was much more positive (65\%), demonstrating that their teaching activities as part of a team had instigated them to look at group performance rather than at individuals. Notably, the fact that in all four high schools at least one positive isolate was obtained created a triumphant atmosphere among the SWITAs. Thus, it seems crucial to emphasize to the high school students the collaborative nature of the experiment and that achievement of individual success is a rare event. On the other hand, a negative individual result may also stimulate social group behavior, and anyhow teaches students persistence and to cope with the frustration of failing experiments, which is key to be a good scientist. It is also important that the students understand that what really matters is what they have learned and that they convey their new knowledge about AMR to friends and family, in that way developing citizenship.

As best aspect of the program, high school students mentioned: the whole program (35\%), the experimental part (44\%), antibiotic discovery (16\%) and interaction with SWITAs (15\%). As the sessions were programmed after school, sometimes coinciding with exam periods, the time schedule was most mentioned as worst or improvable aspect. Other 'critics' involved the short duration of the program, length of theoretical explanations (12\%) and negative results (6\%). In turn, SWITAs best valued the interaction with scholars and teachers (56\%), the teaching 




Figure 3. Phylogenetic neighbor-joining tree showing evolutionary relationships between antibiotic-producing bacteria isolated in this study. The percentage of replicate trees in which the associated taxa clustered together in the bootstrap test (1000 replicates) is shown next to the branches. Evolutionary distances, computed using the Maximum Composite Likelihood method, are in the units of the number of base substitutions per site. Streptomyces griseus NBRC 15744 (NR_112475.1), producer of streptomycin, and Amycolaptosis mediterranei strain NRRL B-3240 (NR_115160.1), producer of rifamycin B, were added as reference species to the alignment. Escherichia coli JCM 1649 (NR_112558.1) was added as outgroup for tree rooting.

opportunity (43\%), learning and experimenting (26\%), addressing a real problem $(22 \%)$ and working in a team (17\%).

In a review analyzing the implementation of service learning in medical education based on 18 studies, a four-stage framework consisting of (i) planning and preparation, (ii) action, (iii) reflection and demonstration and (iv) assessment and celebration was sketched (Stewart and Wubbena 2014). Obviously, dedicated time is especially crucial for the first-time implementation of service learning. Our strategy to create SWITA teams with equal experience by mixing students from different grades and faculties was appreciated by most (65\%) SWITAs but made scheduling the program in the middle of the second semester more complex. Consequently, the timetable was also the most mentioned (35\%) critical point among the SWITAs, and some suggested making future SWITA teams per faculty, which would be easier to organize. Because of the scheduling complexity, we opted not to burden SWITAs with tasks concerning preparation of materials for the high school sessions. However, more than half of the SWITAs answered positively to the question if they think that this would have been advantageous to understand the protocol. When we will run the program again this is something that we plan to incorporate. Sixteen SWITAs had not followed any practical microbiology course prior to the SWI program. It is therefore understandable that not all students found the training sessions sufficient to be able to act as teaching assistants in a microbiology course. Several SWITAs (26\%) also commented that testing and implementing the changes to the antibiosis experiment made it slightly more difficult for them to prepare their classes. In part, this also may be due to the fact that, when starting up this pilot in Albacete, introductory lectures based on the original SWI@Spain protocol were shown to the SWITAs, which may have been slightly confusing.

\section{Preliminary taxonomic analysis}

Preliminary taxonomic analysis of 16S rDNA amplicons of the 23 positive isolates distinguished 17 different bacterial isolates from 13 different soil samples, and 13 different $16 \mathrm{~S}$ rDNA sequences in total (Table 1). Twelve sequences are identical or highly similar to different Streptomyces species, and the remaining one is highly similar to Amycolatopsis species (Table 1; Fig. 3), thus all specify actinomycetes. This confirms the proof-of-principle that our protocol is efficient toward isolating antibiotic-producing actinomycetes and therefore may prove a very beneficial approach in student-sourcing or other programs that are aimed at isolating antibiotic-producing bacteria from soil. We consider a definitive identification, for instance by multi-locus sequence analysis (MLSA) or whole genome sequencing (Labeda et al. 2017; Gupta 2019), of the 23 isolates beyond the scope of this work. For the same reason, we also have not studied whether any of the 23 isolates is actually producing a novel antibiotic compound. Nevertheless, it is noteworthy that sequences of three of the isolates (AB-99-06-10, AB-14-06-04 and $A B-14-07-03)$ were not fully identical to any $16 \mathrm{~S}$ rDNA sequence in the NCBI database, suggesting that our protocol also allows finding novel antibiotic-producing actinomycetes.

The majority of the antibiotics obtained from actinomycetes, for instance streptomycin, neomycin and chloramphenicol, originate from Streptomyces species (Berdy 2005; Barka et al. 2016). Therefore, it is not surprising that most antibiotic producers picked up in this study belong to this genus. Amycolatopsis is also a genus that includes many antibiotic-producing species such as A. mediterranei (producer of rifamycin B) and A. orientalis (vancomycin). We did not encounter antibiotic-producing isolates from other actinomycete genera that include important antibiotic producers (Berdy 2005). As many actinomycete species produce the same antibiotics and identification of new compounds requires enormous screening efforts (Wright 2015), further adaptations of actinomycete isolation protocols (Agate and Bhat 1963) should be considered to improve diversity. For example, considering that actinomycetes are sporulated, growth of non-sporulated bacteria may be inhibited by pre-incubation at $110^{\circ} \mathrm{C}$ for $10 \mathrm{~min}$, and it may be advisable to dry plates at $37^{\circ} \mathrm{C}$ before inoculation to prevent growth of molds. To avoid duplicities in the selection of isolates, colonies can be selected with the aid of a microscope and from different dilutions, including the first dilutions, to choose bacteria that are less abundantly present in the sample.

In conclusion, by changing growth media, incubation conditions and the antibiosis experiment, we have designed a 
modified SWI protocol that is efficient toward isolating antibiotic-producing actinomycetes from soil. The increased rates of positive isolates make citizen science and servicelearning programs aimed at AMR awareness and antibiotic discovery more interesting for the participating students, thereby creating a more effective educational environment.

\section{SUPPLEMENTARY DATA}

Supplementary data are available at FEMSLE online.

\section{ACKNOWLEDGMENTS}

We thank all SWITAs from UCLM and all scholars and teachers from high schools Leonardo da Vinci, Los Olmos, Ramón y Cajal and Universidad Laboral for their participation in the program. We are grateful to Diego Pérez and Nieves Carralero of the 'Dirección Provincial de Educación, Cultura y Deportes' in Albacete for facilities provided. We thank Carmina Rodríguez from the Universidad Complutense de Madrid for providing strains and protocols, and Francisca Vicente and Ignacio González of Fundación Medina for recommendations on working with actinomycetes. We thank SWI and TE managers in the USA, especially Jo Handelsman, Debra Davis and Nichole Broderick, for support along the development of this project.

Conflict of interest. None declared.

\section{REFERENCES}

Abrahamsen L. Learning partnerships between undergraduate biology students and younger learners. Microbiol Educ 2004;5:21-9.

Agate AD, Bhat JV. A method for the preferential isolation of actinomycetes from soils. Antonie Van Leeuwenhoek 1963;29:297304.

Barka EA, Vatsa P, Sanchez L et al. Taxonomy, physiology, and natural products of actinobacteria. Microbiol Mol Biol Rev 2016;80:1-43.

Berdy J. Bioactive microbial metabolites. J Antibiot 2005;58:1-26.

Bringle RG, Hatcher JA. Implementing service learning in higher education. J High Educ 1996;67:221.

Butler MS, Blaskovich MA, Cooper MA. Antibiotics in the clinical pipeline at the end of 2015. J Antibiot 2017;70:3-24.
Butler MS, Cooper MA. Antibiotics in the clinical pipeline in 2011. J Antibiot 2011;64:413-25.

Cantón R, Morosini MI. Emergence and spread of antibiotic resistance following exposure to antibiotics. FEMS Microbiol Rev 2011;35:977-91.

Caruso JP, Israel N, Rowland $\mathrm{K}$ et al. Citizen Science: the Small World Initiative improved lecture grades and California Critical Thinking Skills Test scores of nonscience major students at Florida Atlantic University. J Microbiol Biol Educ 2016;17:15662.

DeLuca M, King R, Morsy M. Bioprospecting saline gradient of a wildlife sanctuary for bacterial diversity and antimicrobial activities. BMC Res Notes 2017;10:397.

Emmert EA. Biosafety guidelines for handling microorganisms in the teaching laboratory: development and rationale. $J$ Microbiol Biol Educ 2013;14:78-83.

Gupta RS. Commentary: genome-based taxonomic classification of the phylum actinobacteria. Front Microbiol 2019;10: 206.

Hernandez S, Tsang T, Bascom-Slack C et al. Tiny Earth-A Research Guide to Studentsourcing Antibiotic Discovery, 18th edn. Ann Arbor, MI: XanEdu Publishing Inc., 2018.

Kumar S, Stecher G, Li M et al. MEGA X: molecular evolutionary genetics analysis across computing platforms. Mol Biol Evol 2018;35:1547-9.

Labeda DP, Dunlap CA, Rong X et al. Phylogenetic relationships in the family Streptomycetaceae using multi-locus sequence analysis. Antonie Van Leeuwenhoek 2017;110: 563-83.

Leung E, Weil DE, Raviglione $\mathrm{M}$ et al. The WHO policy package to combat antimicrobial resistance. Bull World Health Organ 2011;89:390-2.

Stewart T, Wubbena Z. An overview of infusing service learning in medical education. Int J Med Educ 2014;5:147-56.

Valderrama MJ, Gonzalez-Zorn B, de Pablo PC et al. Educating in antimicrobial resistance awareness: adaptation of the Small World Initiative program to service learning. FEMS Microbiol Lett 2018;365:fny161.

Webb G. A review of microbiology service learning. FEMS Microbiol Lett 2017;364:fnx032.

Weisburg WG, Barns SM, Pelletier DA et al. 16S ribosomal DNA amplification for phylogenetic study. J Bacteriol 1991;173:697703.

Wright GD. Solving the antibiotic crisis. ACS Infect Dis 2015;1: 80-4. 